\title{
Polypille einmal täglich gegen Herzinfarkt und Schlaganfall
}

\section{Eine Tablette mit Kalziumantago- nist, Sartan, Diuretikum und Statin könnte sich bei Personen ab dem 50. Lebensjahr in der Primärprävention kardiovaskulärer Erkrankungen als sehr erfolgreich erweisen.}

- In einer randomisierten, doppelblinden Crossover-Studie erhielten 84 mindestens 50 Jahre alte Probanden (im Mittel 59 Jahre) ohne kardiovaskuläre Vorerkrankungen für die Dauer von zwölf Wochen nach Zufallskriterien zuerst die Polypille mit 2,5 mg Amlodipin, $25 \mathrm{mg}$ Losartan, 12,5 mg Hydrochlorothiazid und $40 \mathrm{mg}$ Simvastatin einmal abends und dann Placebo oder in umgekehrter Reihenfolge. Eine evtl. Vorbehandlung der Patienten wurde zu Studienbeginn abgesetzt. Am Ende der Placeboperioden lag der Blutdruck bei 143/86 $\mathrm{mmHg}$ und das LDL bei $143 \mathrm{mg} / \mathrm{dl}$ (3,7 $\mathrm{mmol} / \mathrm{l})$.

Im Vergleich zu den Placeboperioden fielen unter der Polypille der Blutdruck absolut um 17,9 mmHg systolisch, 9,8 diastolisch und das LDL-Cholesterin um $54 \mathrm{mg} / \mathrm{dl}(1,4 \mathrm{mmol} / \mathrm{l})$, relativ um 12, 11 und $39 \% \mathrm{ab}$. Diese Veränderungen waren praktisch identisch mit denen, die aus Studien mit den vier Ein-

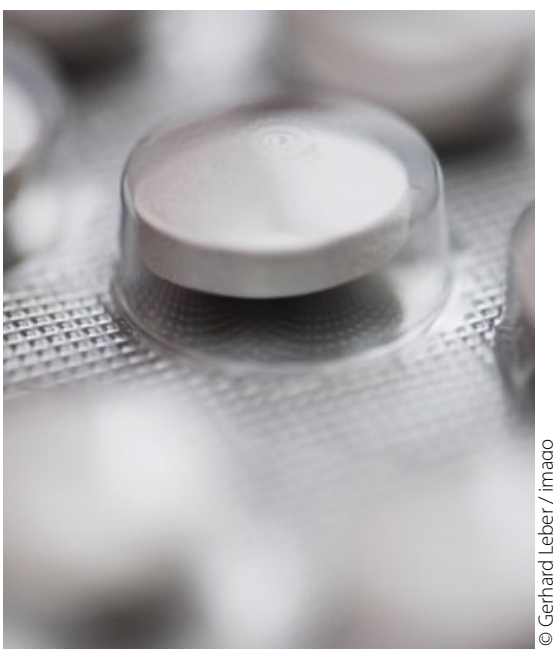

Radikales Konzept: Polypille für alle ÜberFünfzigjährigen.

zelkomponenten errechnet wurden, nämlich 18,4/9,7 mmHg bzw. $54 \mathrm{mg} / \mathrm{dl}$. 98\% der Probanden nahmen mehr als $85 \%$ der verordneten Tabletten. Nur elf Patienten berichteten über Nebenwirkungen, die aber nicht zum Abbruch der Einnahme führten.

\section{Kommentar}

Das einzige Einschlusskriterium für diese Studie war ein Alter von mindestens 50 Jahren. Die Probanden konnten, mussten aber keine
Hypertonie und keine Hyperlipidämie haben, trotzdem erhielten sie drei Antihypertensiva und ein Statin. Da alle Versuchspersonen noch keine Herz-Kreislauf-Erkrankungen hatten, handelt es sich um eine Studie zur Primärprävention. Bei großer Zuverlässigkeit der Einnahme und sehr geringen Nebenwirkungen waren die Senkungen von Blutdruck und Lipiden erheblich. Daraus kann man eine Abnahme von koronaren Komplikationen um $72 \%$ und von Schlaganfällen um $64 \%$ vorhersagen. Oder anders formuliert: $28 \%$ aller Personen, die ab dem 50. Lebensjahr die Polypille nehmen, können zeitlebens einen Herzinfarkt vermeiden und ihr Leben um elf Jahre verlängern, ohne eine kardiovaskuläre Erkrankung zu erleben. ASS wurde zwar als weitere Komponente in der Polypille erwogen, doch wegen des Blutungsrisikos bei noch herzkreislaufgesunden Probanden nicht akzeptiert.

Das Konzept der kardiovaskulären Primärprävention durch die Polypille ab dem 50. Lebensjahr hat aber auch Kritiker: Das Konzept gilt als zu radikal. Viele Experten empfehlen weiterhin eine individuelle Bestimmung und gezielte Behandlung von Risikofaktoren.

H. HOLZGREVE —

D. S. Wald et al.

Randomized polypill crossover trial in people aged 50 and over. Plos One 2012; 7 (7): e41297 